\title{
Parity-Violating Nuclear Force as derived from QCD Sum Rules
}

\author{
W-Y. P. Hwang and Chih-Yi Wen \\ The Leung Research Center for Cosmology and Particle Astrophysics, \\ Center for Theoretical Sciences, \\ Department of Physics, and Institute of Astrophysics, \\ National Taiwan University, \\ Taipei, Taiwan 106, ROC
}

January 26, 2008

\begin{abstract}
Parity-violating nuclear force, as may be accessed from parity violation studies in nuclear systems, represents an area of nonleptonic weak interactions which has been the subject of experimental investigations for several decades. In the simple meson-exchange picture, parity-violating nuclear force may be parameterized as arising from exchange of $\pi, \rho, \omega$, or other meson(s) with strong meson-nucleon coupling at one vertex and weak parity-violating meson-nucleon coupling at the other vertex. The QCD sum rule method allows for a fairly complicated, but nevertheless straightforward, leading-order loop-contribution determination of the various parity-violating $M N N$ couplings starting from QCD (with the nontrivial vacuum) and Glashow-Salam-Weinberg electroweak theory. We continue our earlier investigation of parity-violating $\pi N N$ coupling (by Henley, Hwang, and Kisslinger) to other parity-violating couplings. Our predictions are in reasonable overall agreement with the results estimated on phenomenological grounds, such as in the now classic paper of Desplanques, Donoghue, and Holstein (DDH), in the global experimental fit of Adelberger and Haxton $(\mathrm{AH})$, or the effective field theory (EFT) thinking of RamseyMusolf and Page (RP).
\end{abstract}

PACS: 24.80.+y (Nuclear tests of fundamental interactions and symmetries), 24.85.+p (Quarks, gluons, and QCD in nuclei and nuclear processes). 


\section{Introduction}

Parity violation (PV) studies in nuclear systems, such as the asymmetry[1, 2] in $\vec{p}+p \rightarrow p+p$, the photon circular polarization measurement in $n+p \rightarrow d+\gamma$, and PV studies in ${ }^{18} \mathrm{~F}$ and ${ }^{21} \mathrm{Ne}$, offer a means of determining the parity-violating nuclear force, which represents a special category of nonleptonic weak interactions accessible experimentally. In the simple meson-exchange picture, parity-violating nuclear force arises from exchange of $\pi, \rho, \omega$, or other meson(s) with strong $M N N$ coupling at one vertex and weak parity-violating $M N N$ coupling at the other vertex. In 1980's, parity-violating meson-nucleon couplings have been estimated primarily on phenomenological grounds, such as in the classic paper of Desplanques, Donoghue, and Holstein (DDH) [3]. A global fit to obtain these PV meson-nucleon couplings, making use of the various experimental data available at the time, was performed also (in 1985) by Adelberger and Haxton (AH) [4. Nevertheless, progress [5, both experimental and theoretical, has been slow since then, although the interest in the problem arose to some extent in view of the adopted effective field theory for the few nucleon systems.

In 1980's, the standard model of particle physics, which consists of GlashowSalam-Weinberg [GSW] electroweak theory and quantum chromodynamics [QCD], had meanwhile been well established. Although the nonperturbative feature of QCD manifests itself in formation of hadron structure and makes it very difficult to predict quantitatively hadron properties including both the strong parity-conserving and weak parity-violating meson-nucleon couplings, the method of QCD sum rules [6] offers a systematic way for taking into account effects as caused by the nontrivial nature of the QCD ground state or the QCD vacuum. The QCD sum rule method has become quite popular starting from 1990's (till now). In view of the laborious efforts in lattice gauge theory, the QCD sum rule method does offer an alternative avenue to obtain the various predictions, to some extent in the spirit of QCD.

To "complete" the study of nuclear parity violations, we need to investigate both the parity-conserving and parity-violating meson-nucleon couplings, using the conventional scheme of nuclear forces. As already shown in another paper [7, it is possible to use the method of QCD sum rules in external fields [8] to determine the strong $\pi N N, \rho N N$, and $\omega N N$ couplings. The purpose of this paper is to present a QCD sum rule determination of the parity-violating $\rho N N$ and $\omega N N$ couplings, which turns out to be fairly complicated but nevertheless straightforward. Our present results, together with a previous study of the parity-violating $\pi N N$ coupling [10], allow for a direct connection between parity-violating nuclear force and the standard model, despite the complication of QCD.

The external-field QCD sum rule method [8] have been used to treat the strong $\pi N N$ coupling [11], the weak parity-violating $\pi N N$ coupling [10], and the strong $\rho N N$ and $\omega N N$ couplings [7]. In all cases considered, quantitative successes have been achieved mainly because the nonperturbative effects of QCD, as expressed in terms 
of induced condensates, have been taken into account and are found to be of critical importance. Our efforts to treat hyperon weak decays remain in progress [12], with fairly encouraging results (which are beyond the scope of the present article). Therefore, it is natural to follow the same method to treat the weak parity-violating $\rho N N$ and $\omega N N$ couplings. Nevertheless, it remains difficult to assess properly whether our present calculation may also succeed in a quantitative manner, since after all the nonleptonic amplitudes in question are "notoriously difficult to calculate" (quoting the phrase from the referee on the early version of one of my early articles). However, we have good reasons, based on successful experiences on the method of QCD sum rules in general, to believe that the complicated task carried out in this research could be the first important step in establishing a benchmark in the (future) quantitative treatments of this difficult problem. Just like many other nonleptonic weak decays where relevant data exist, we already have experimental information [4, 5] which we may use to test our theoretical predictions.

For the sake of completeness, we begin by outlining a few ingredients regarding the external-field QCD sum rule method, without detailed qualifying statements. The external vector field is expressed as

$$
Z_{\mu}=-\frac{1}{2} Z_{\mu \nu} x^{\nu}
$$

We attempt to determine the following polarization function for a nucleon in a small (classical) external vector-meson field $Z_{\mu}$ (where $Z$ represents either a $\rho$ or $\omega$ meson):

$$
i \int d^{4} x e^{i q \cdot x}\left\langle 0\left|T \eta_{N}(x) \bar{\eta}_{N}(0)\right| 0\right\rangle_{Z}=\Pi(q)+h Z_{\mu \nu} \Pi^{\mu \nu}(q),
$$

where the numerical constant $h$ is the coupling between the external field and the up $(u)$ quark field; more explicitly, the coupling between the external field and the down $(d)$ quark is $-h$ for $\rho$ meson and $+h$ for $\omega$ meson. The standard form for the composite operator $\eta_{N}(x)$ is adopted [9]:

$$
\begin{aligned}
& \eta_{p}(x)=\epsilon^{a b c}\left[u^{a T}(x) C \gamma_{\mu} u^{b}(x)\right] \gamma_{5} \gamma^{\mu} d^{c}(x), \\
& \eta_{n}(x)=\epsilon^{a b c}\left[d^{a T}(x) C \gamma_{\mu} d^{b}(x)\right] \gamma_{5} \gamma^{\mu} u^{c}(x),
\end{aligned}
$$

which transform like the proton and neutron fields, respectively. Here $u^{a}(x)$ and $d^{a}(x)$ are the up and down quark fields with the superscript $a$ the color index and $C$ is the charge conjugation operator.

At the hadronic level, we define the parity-violating meson-nucleon couplings related to $\rho$ and $\omega$ mesons in the standard manner 3 .

$$
\begin{aligned}
L_{i n t}^{p . v .}= & -\bar{N}\left[h_{\rho}^{0} \vec{\tau} \cdot \vec{\phi}_{\mu}^{\rho}+h_{\rho}^{1} \phi_{\mu}^{\rho 3}+\frac{h_{\rho}^{2}}{2 \sqrt{6}}\left(3 \tau^{3} \phi_{\mu}^{\rho 3}-\vec{\tau} \cdot \vec{\phi}_{\mu}^{\rho}\right)\right] \gamma^{\mu} \gamma_{5} N \\
& +h_{\rho}^{\prime} \bar{N}\left(\vec{\tau} \times \vec{\phi}_{\mu}^{\rho}\right)^{3} \frac{\sigma^{\mu \nu} k_{\nu}}{2 m} \gamma_{5} N-\bar{N}\left[h_{\omega}^{0} \phi_{\mu}^{\omega}+h_{\omega}^{1} \tau^{3} \phi_{\mu}^{\omega}\right] \gamma^{\mu} \gamma_{5} N .
\end{aligned}
$$


Here the superscripts in the couplings $h_{\rho}^{0}, h_{\rho}^{1}, h_{\rho}^{2}, h_{\rho}^{\prime 1}, h_{\omega}^{0}$, and $h_{\omega}^{1}$ refer to the isospin character as the weak interactions do not observe isospin symmetry. We may use the above expression to write down the polarization function $\Pi(q)$, with the paricular piece which is proportional to $h$, at the hadronic level. It is clear that we need to combine different channels (i.e. $p \rightarrow p, n \rightarrow n, p \rightarrow n$, and $n \rightarrow p$ ) in order to extract these PV coupling constants.

At the quark level, we use weak interactions as described by the Glashow-WeinbergSalam (GSW) electroweak theory to determine the polarization function, obtaining in general three-loop diagrams which require regularizations. Here we adopt dimensional regularization in the minimum-subtraction (MS) scheme and introduce suitable counter terms in defining the renormalized operators $\left[\eta_{p}\right]_{R}$ and $\left[\eta_{n}\right]_{R}$. As mentioned above, we may parametrize the same polarization function phenomenologically, making use of the parity-violating $\rho N N$ and $\omega N N$ interaction Lagrangians. Comparing the results obtained through the two ways of evaluating the polarization functions (i.e., at the quark level using GSW theory and QCD and at the hadronic level involving $\rho N N$ and $\omega N N$ couplings), we have a definitive way in extracting the weak PV $\rho N N$ and $\omega N N$ couplings - the primary objective of this paper.

\section{The parity-violating $\rho N N$ and $\omega N N$ couplings}

As a special feature in relation to the method of QCD sum rules, the kinematic variable $q_{\mu}^{2}$ of the polarization function $\Pi(q)$, as translated into the choice of the Borel mass squared $M^{2}$, which is in the vicinity of slightly above $1 \mathrm{GeV}^{2}$. Such choice of the Borel mass is to ensure the approximate validity of the operator-product expansion (OPE) augmented with power corrections (as due to the various condensates). In other words, perturbative QCD corrections to the coefficients in such OPE are in principle there but are presumably suppressed by choice of the Borel mass $M$. Unlike what has been involved in most phenomenological approaches to the problem where some effective weak Hamiltonian at the energy scale relevant to the hadron must be directly invoked, we have in the QCD sum rule method the nice feature that the GSW electroweak theory is called for at the scale set by the Borel mass squared $M^{2}$ where effects to order $O\left(G_{F} \alpha_{S}\right)$ are suppressed (due to the running of the strong coupling $\left.\alpha_{S}\right)$ and it is the intrinsic smooth extrapolation of the results to lower $q^{2}$ which helps to explain the successes of the predictions.

Accordingly, it should be possible to use, in the QCD sum rule method, the effective GSW electroweak lagrangian at tree level while leaving terms in $O\left(G_{F} \alpha_{S}\right)$ as corrections. That is, we may use, as a good starting point,

$$
\begin{aligned}
L_{\text {weak }}= & -\frac{G_{F}}{\sqrt{2}}\left[\bar{u} \gamma_{\mu}\left(1-\gamma_{5}\right) d \bar{d} \gamma^{\mu}\left(1-\gamma_{5}\right) u+\right. \\
& \left.\sum_{q_{1}, q_{2}=u, d} \bar{q}_{1} \gamma_{\mu}\left(A_{q_{1}}-B_{q_{1}} \gamma_{5}\right) q_{1} \bar{q}_{2} \gamma^{\mu}\left(A_{q_{2}}-B_{q_{2}} \gamma_{5}\right) q_{2}\right]
\end{aligned}
$$


where $G_{F}$ is the Fermi coupling constant $\left(G_{F}=1.166 \times 10^{-5} \mathrm{GeV}^{-2}\right)$ and the other constants are defined through $A^{u}=\frac{1}{2}-\frac{4}{3} \sin ^{2} \theta_{W}, B^{u}=\frac{1}{2}, A^{d}=-\frac{1}{2}+\frac{2}{3} \sin ^{2} \theta_{W}$ and $B^{d}=-\frac{1}{2}$ with $\sin ^{2} \theta_{W}$ the electroweak mixing parameter $\left(\sin ^{2} \theta_{W}=0.2315\right)$. Note that the first term in the above equation comes from exchange of the $W^{ \pm}$boson while the second term is due to $Z^{0}$ exchange. In view of our primary goal which is to identify the sizable role played by the various condensates, it is clear that the issue regarding the renormalization of the tree lagrangian (as would be suppressed by the choice of the Borel mass) is of secondary importance and could be addressed when higher precision (in theoretical prediction) is called for. As shall be explained later, it is in fact sufficient to even use the current-current form since the difference from what we may obtain by employing the renormalizable gauge throughout, such as the $R_{\xi}$ gauge, does not matter at the end as far as our final QCD sum rules are concerned.

\subsection{Renormalization of composite operators}

Inclusion of the weak interaction in the polarization function leads to sub-divergences even in the lowest order $O\left(G_{F}\right)$. Such divergences may be removed (or regularized) by making use of a suitably defined renormalized composite operators $[\eta]_{R}$ and $[\bar{\eta}]_{R}$. Such renormalized operators may be obtained by considering the four-fermion interaction:

$$
\begin{aligned}
& \int d^{d} x d^{d} y e^{i(p \cdot x+q \cdot y)}\left\langle u_{i^{\prime}}^{a^{\prime}}(0) d_{j^{\prime}}^{b^{\prime}}(0) \bar{u}_{i}^{a}(x) \bar{d}_{j}^{b}(y)\right\rangle=\frac{\delta^{a^{\prime} a} \delta^{b^{\prime} b}}{p^{2} q^{2}} \hat{p}_{i^{\prime} i} \hat{q}_{j^{\prime} j}+ \\
& \frac{G_{F}}{\sqrt{2}} \frac{1}{48 \pi^{2}(d-4)} \frac{1}{p^{2} q^{2}}\left[(p+q)_{\rho}(p+q)_{\sigma}+\frac{1}{2}(p+q)^{2} g_{\rho \sigma}\right] \times \\
&\left\{\left[\gamma^{\rho} \gamma_{\mu}\left(A^{u}-B^{u} \gamma_{5}\right) \hat{p}\right]_{i^{\prime} i}\left[\gamma^{\sigma} \gamma^{\mu}\left(A^{d}-B^{d} \gamma_{5}\right) \hat{q}\right]_{j^{\prime} j}+\right. \\
& {\left.\left[\gamma^{\rho} \gamma_{\mu}\left(1-\gamma_{5}\right) \hat{p}\right]_{i^{\prime} i}\left[\gamma^{\sigma} \gamma^{\mu}\left(1-\gamma_{5}\right) \hat{q}\right]_{j^{\prime} j}\right\}+ \text { finite parts } }
\end{aligned}
$$

where the first term is the free piece while the second term, which is divergent at $d=4$, comes from the insertion of the weak interaction. This equation enables us to define the renormalized operator $[u(x) d(x)]_{R}$. Using the minimum-subtraction (MS) scheme, we obtain the renormalized operator $[u(x) d(x)]_{R}$ as follows:

$$
\begin{aligned}
{\left[u_{i}^{a}(x) d_{j}^{b}(x)\right]_{R}=} & u_{i}^{a}(x) d_{j}^{b}(x)+\frac{G_{F}}{\sqrt{2}} \frac{\mu^{d-4}}{48 \pi^{2}(d-4)}\left(\partial_{\rho} \partial_{\sigma}+\frac{1}{2} g_{\rho \sigma} \sqcup\right) \times \\
& \left\{\left[\gamma^{\rho} \gamma_{\mu}\left(A^{u}-B^{u} \gamma_{5}\right) u^{a}(x)\right]_{i}\left[\gamma^{\sigma} \gamma^{\mu}\left(A^{d}-B^{d} \gamma_{5}\right) d^{b}(x)\right]_{j}+\right. \\
& {\left.\left[\gamma^{\rho} \gamma_{\mu}\left(1-\gamma_{5}\right) u^{a}(x)\right]_{i}\left[\gamma^{\sigma} \gamma^{\mu}\left(1-\gamma_{5}\right) d^{b}(x)\right]_{j}\right\} . }
\end{aligned}
$$

Here and what as follows, the notations are defined in accord with the previous equation such as $\left(\partial_{\rho} \partial_{\sigma}+\frac{1}{2} g_{\rho \sigma} \bar{\sqcup}\right)$ as coming from $\left[(p+q)_{\rho}(p+q)_{\sigma}+\frac{1}{2}(p+q)^{2} g_{\rho \sigma}\right]$. Similarly, the renormalized operators $[u(x) u(x)]_{R}$ and $[d(x) d(x)]_{R}$ are given by 


$$
\begin{aligned}
{\left[u_{i}^{a}(x) u_{j}^{b}(x)\right]_{R}=} & u_{i}^{a}(x) u_{j}^{b}(x)+\frac{G_{F}}{\sqrt{2}} \frac{\mu^{d-4}}{48 \pi^{2}(d-4)}\left(\partial_{\rho} \partial_{\sigma}+\frac{1}{2} g_{\rho \sigma} \square\right) \times \\
& {\left[\gamma^{\rho} \gamma_{\mu}\left(A^{u}-B^{u} \gamma_{5}\right) u^{a}(x)\right]_{i}\left[\gamma^{\sigma} \gamma^{\mu}\left(A^{u}-B^{u} \gamma_{5}\right) u^{b}(x)\right]_{j} . } \\
{\left[d_{i}^{a}(x) d_{j}^{b}(x)\right]_{R}=} & d_{i}^{a}(x) d_{j}^{b}(x)+\frac{G_{F}}{\sqrt{2}} \frac{\mu^{d-4}}{48 \pi^{2}(d-4)}\left(\partial_{\rho} \partial_{\sigma}+\frac{1}{2} g_{\rho \sigma} \square\right) \times \\
& {\left[\gamma^{\rho} \gamma_{\mu}\left(A^{d}-B^{d} \gamma_{5}\right) d^{a}(x)\right]_{i}\left[\gamma^{\sigma} \gamma^{\mu}\left(A^{d}-B^{d} \gamma_{5}\right) d^{b}(x)\right]_{j} . }
\end{aligned}
$$

The renormalized composite operators $\left[\eta_{n}\right]_{R}$ and $\left[\eta_{p}\right]_{R}$ may now be deduced directly from the above three equations. This corresponds to introduction of the counter terms which cancel the subdivergences. We find that $[\eta]_{R}$ is given by

$$
\begin{aligned}
{\left[\eta_{p}\right]_{R}=} & \epsilon^{a b c}\left[u^{a T}(x) C \gamma_{\mu} u^{b}(x)\right] \gamma_{5} \gamma_{\mu} d^{c}(x)+\frac{G_{F}}{\sqrt{2}} \frac{\mu^{d-4} \epsilon^{a b c}}{24 \pi^{2}(d-4)} \times \\
& \left(\partial_{\rho}^{y} \partial_{\sigma}^{y}+\frac{1}{2} g_{\rho \sigma}(\bar{\sqcup})_{y}\right) \times\left\{-\left[u^{a T}(y) C \gamma^{\sigma} \gamma_{\mu} \gamma^{\rho} u^{b}(y)\right] \gamma_{5} \gamma_{\mu} d^{c}(x)\right. \\
& +\left[u^{a T}(x) C \gamma_{\mu} \gamma^{\rho} \gamma_{\nu}\left(A^{u}-B^{u} \gamma_{5}\right) u^{b}(y)\right] \gamma_{5} \gamma_{\mu} \gamma^{\sigma} \gamma^{\nu}\left(A^{d}-B^{d} \gamma_{5}\right) d^{c}(y) \\
& \left.+\left[u^{a T}(x) C \gamma_{\mu} \gamma^{\rho} \gamma_{\nu}\left(1-\gamma_{5}\right) u^{b}(y)\right] \gamma_{5} \gamma_{\mu} \gamma^{\sigma} \gamma^{\nu}\left(1-\gamma_{5}\right) d^{c}(y)\right\}\left.\right|_{y=x}
\end{aligned}
$$

An analogous expression for $\left[\eta_{n}\right]_{R}$ may be obtained by exchanging all the $u$ 's and $d$ 's. We note that, once the subdivergences are removed in this way, the overall divergence (associated with the polarization function) becomes local and thus is removed upon Borel transform (as employed in the context of QCD sum rules). The difference our result from what we may obtain by working throughout with the renormalizable $R_{\xi}$ gauge also disappears upon Borel transformation.

\subsection{QCD sum rules for the parity-violating couplings}

The various diagrams which we need to consider are illustrated in Figs. 1, where the propagator with a thick external-line mark is to be understood as the quark propagator in the presence of an external vector field. [7, 8. At the quark level, we calculate the polarization function by considering only the leading two terms in the operator product expansion - the first one is associated with the operator $\langle 1\rangle$ and the second with the operator $<\bar{q} \sigma_{\mu \nu} q>$. As explained immediately below, the resultant expressions are already extremely complicated algebraically, preventing 

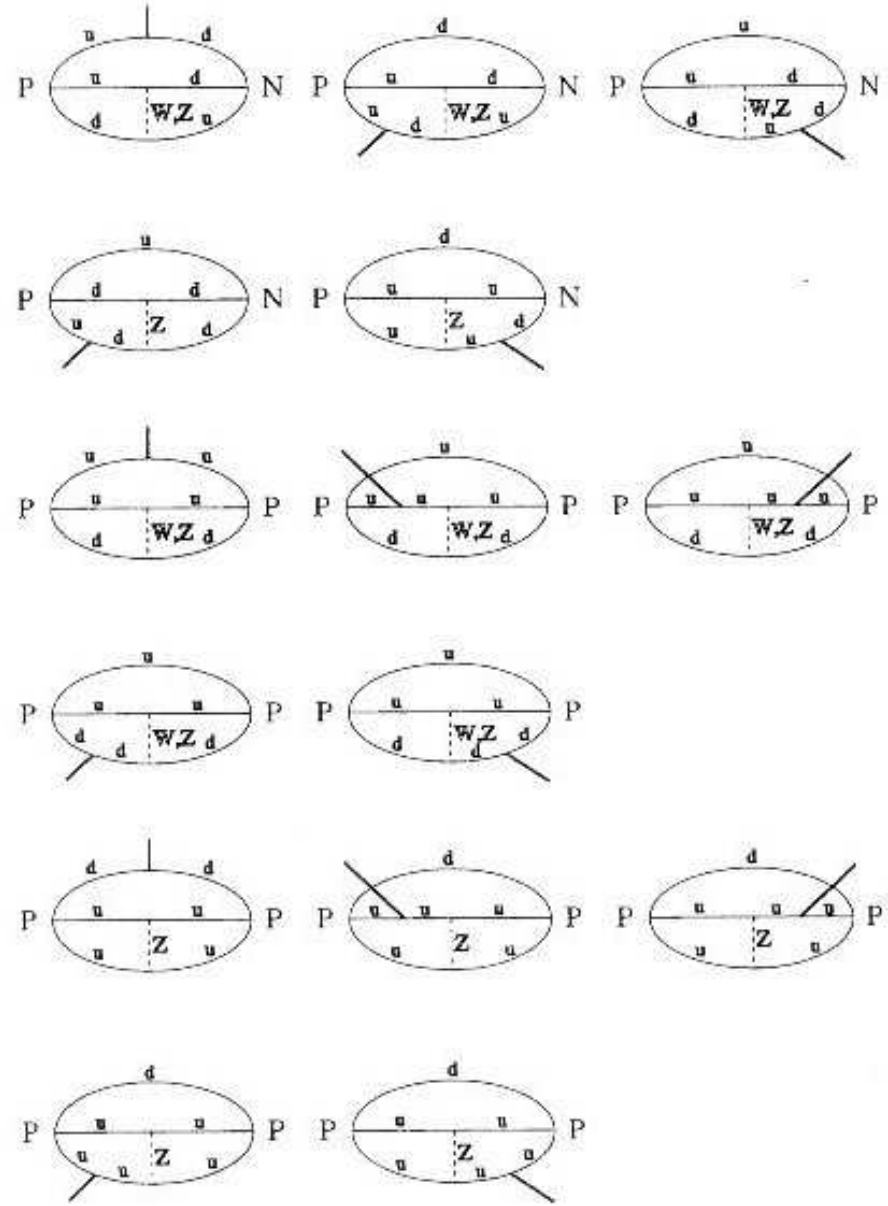

Figure 1: Loop diagrams for PV-coupling sum-rule calculations.

us from carrying out a better and more complete calculation (unless a considerable amount of time is invested).

To get a better feeling towards the extensiveness of the problem, we may look at the following expression which is associated with the coefficient of the operator $<1>$ :

$$
\begin{aligned}
& \int \frac{d^{d} p_{1}}{(2 \pi)^{d}} \frac{d^{d} p_{2}}{(2 \pi)^{d}} \frac{d^{d} p_{3}}{(2 \pi)^{d}} \frac{p_{1}^{a} p_{2}^{b}\left(q-p_{3}\right)^{c}\left(p_{3}-p_{1}\right)^{d}\left(p_{3}-p_{2}\right)^{e}}{p_{2}^{2}\left(q-p_{3}\right)^{4}\left(p_{1}-p_{3}\right)^{2}\left(p_{2}-p_{3}\right)^{2}} \times \\
& \gamma^{\mu} \gamma_{a} \gamma_{\alpha}\left(A^{d}-B^{d} \gamma_{5}\right) \gamma_{b} \gamma_{\nu}\left(\gamma_{c} \sigma_{\rho \sigma}+\sigma_{\rho \sigma} \gamma_{c}\right) \gamma_{\mu} \gamma_{d} \gamma_{\alpha}\left(A^{u}-B^{u} \gamma_{5}\right) \gamma_{e} \gamma^{\nu},
\end{aligned}
$$

which involves taking the trace of the product of 13 gamma matrices, a task that needs a good algebraic software package (such as Mathematica). It also involves 
three integration variables $p_{1}, p_{2}$ and $p_{3}$ and dimensional regularization is required during the integration. To work out the problem, we have chosen to simplify these three-loop expressions by devising algebraic programs making use of Mathematica. It still takes up considerable amount of computer time in solving the problem. In practice, we perform the calculation in two steps: First, we do the integrations in the order of $p_{1}$ and $p_{2}$, then $p_{3}$. This needs a liitle program written in Mathematica to handle it (using the various formulae suitable in $d$-dimensions). The result contains about a hundred terms with different tensor structures. Second, we contract the result with the gamma matrices and simplifying it. For this step we use the Mathematica package "FeynCalc 1.0" (written by Rolf Mertig). The final result is

$$
\begin{aligned}
& -\frac{q^{4}}{\pi^{6}}\left(-\hat{q} g_{\rho \sigma}+\hat{q} \gamma_{\rho} \gamma_{\sigma}-\gamma_{\sigma} q_{\rho}+\gamma_{\rho} q_{\sigma}\right)\left[\left(A^{d} A^{u}+B^{d} B^{u}\right)\right. \\
& \left.-\left(A^{u} B^{d}+A^{d} B^{u}\right) \gamma_{5}\right] \times\left\{\frac{1}{384(d-4)^{2}}+\frac{1}{27648(d-4)}\right. \\
& \left.[-275+108 X]+\frac{1}{165888}\left[4345-27 \pi^{2}-2475 X+486 X^{2}\right]\right\}
\end{aligned}
$$

where $X=\gamma-\log (4 \pi)+\log \left(-q^{2}\right)$. The presence of the pole term $X /(d-4)$ indicates that this diagram contains subdivergences. The subdivergences come from the $p_{1}$ and $p_{2}$ integrals. The removal of these subdivergences is done by making use of the renormalized operators $\left[\eta_{p}\right]_{R}$ and $\left[\eta_{n}\right]_{R}$.

We have examined the question of how to define $\gamma_{5}$ in $d$-dimensions, since the definition of $\gamma_{5}$ is a tricky issue in the $d$-dimension ('t Hooft \& Veltman in 1972). In the present case, however, what we may do is to ignore this fact and anti-commute it with all the $\gamma_{\mu}$ 's to reach the utmost right position. This is what we have done in the above expresion, i.e., by moving all the $\gamma_{5}$ matrix to the right of all gamma matrices. After removing all the subdivergences, the difference between using the proper procedure and using the naive method turns out to be only a polynomial of $q^{2}$. The difference does not contain nonlocal terms like $\ln \left(-q^{2}\right)$ or $1 / q^{2}$ which we must handle with care.

At the hadron level, there are four coupling constants for the $\rho$-meson and two for the $\omega$-meson so that the calculation should be carried out for the various channels including $\rho^{+} n p, \rho^{-} p n, \rho^{0} p p, \rho^{0} n n, \omega p p$, and $\omega n n$ and suitable linear combinations allow for the determination of the various PV couplings.

We choose to focus on the antisymmetric part of the sum rules (proportional to $Z_{\mu \nu}^{A}$ with $Z_{\nu \mu}^{A}=-Z_{\mu \nu}^{A}$ ), which already contain enough information to determine the meson-nucleon couplings. Different tensor structures leads to different sum rules. After suitably adding and subtracting between these $\rho N N(\omega N N)$ sum rules we obtain the following results (in the MS scheme). 


$$
\begin{aligned}
\frac{\lambda_{N}^{2} h_{\rho}^{0}}{\left(q^{2}-m^{2}\right)^{2}}= & -\frac{G_{F} h}{\sqrt{2}} \frac{q^{4}}{256 \pi^{6}} \times \\
& \left(31 \ln \left(-\frac{q^{2}}{\bar{\mu}^{2}}\right)-6 \ln ^{2}\left(-\frac{q^{2}}{\bar{\mu}^{2}}\right)\right)\left(\frac{1}{2}+\sin ^{2} \theta_{W}\right) \\
\frac{\lambda_{N}^{2} h_{\rho}^{1}}{\left(q^{2}-m^{2}\right)^{2}}= & \frac{G_{F} h}{\sqrt{2}} \frac{q^{4}}{256 \pi^{6}} \times \\
\frac{\lambda_{N}^{2} h_{\rho}^{1^{\prime}}}{2 m\left(q^{2}-m^{2}\right)^{2}}= & -\frac{1}{36}\left(59 \ln \left(-\frac{q^{2}}{\bar{\mu}^{2}}\right)-6 \ln ^{2}\left(-\frac{q^{2}}{\bar{\mu}^{2}}\right)\right)\left(\frac{1}{3} \sin ^{2} \theta_{W}\right) \\
& \left(13 \ln \left(-\frac{q^{2}}{\bar{\mu}^{2}}\right)-3 \ln ^{2}\left(-\frac{q^{2}}{\bar{\mu}^{2}}\right)\right)\left(\frac{1}{3} \sin ^{2} \theta_{W}\right) \\
\frac{\lambda_{N}^{2} h_{\omega}^{0}}{\left(q^{2}-m^{2}\right)^{2}}= & -\frac{G_{F} h}{\sqrt{2}} \frac{q^{4}}{256 \pi^{6}} \times \\
& \left(31 \ln \left(-\frac{q^{2}}{\bar{\mu}^{2}}\right)-6 \ln ^{2}\left(-\frac{q^{2}}{\bar{\mu}^{2}}\right)\right)\left(\frac{1}{2}+\sin ^{2} \theta_{W}\right) \\
\frac{\lambda_{N}^{2} h_{\omega}^{1}}{\left(q^{2}-m^{2}\right)^{2}}= & -\frac{G_{F} h}{\sqrt{2}} \frac{q^{4}}{256 \pi^{6}} \times \\
& \frac{5}{36}\left(35 \ln \left(-\frac{q^{2}}{\bar{\mu}^{2}}\right)-6 \ln ^{2}\left(-\frac{q^{2}}{\bar{\mu}^{2}}\right)\right)\left(\frac{1}{3} \sin ^{2} \theta_{W}\right) \\
= &
\end{aligned}
$$

where $\ln \left(1 / \bar{\mu}^{2}\right)=\gamma+\ln \left(1 / 4 \pi \mu^{2}\right)$. The entity $\mu$ is the renormalization scale used in dimensional regularization. $\sin ^{2} \theta_{W}$ is the electroweak mixing parameter in the GSW electroweak theory.

Performing Borel transformation on both sides, taking into account anomalous dimensions for the various terms in the OPE, and making use of the continuum approximation for contributions from higher exited states, we find

$$
\begin{aligned}
\frac{\lambda_{N}^{2} h_{\rho}^{0}}{M^{2}} e^{-\frac{m^{2}}{M^{2}}=} & -\frac{G_{F} h}{\sqrt{2}} \frac{M^{6}}{256 \pi^{6}} L^{-\frac{4}{9}} \times \\
& {\left[-62 E_{2}\left(\frac{W}{M}\right)+24 F_{2}\left(\frac{W}{M}, \frac{M}{\bar{\mu}}\right)\right]\left(\frac{1}{2}+\sin ^{2} \theta_{W}\right) } \\
\frac{\lambda_{N}^{2} h_{\rho}^{1}}{M^{2}} e^{-\frac{m^{2}}{M^{2}}=} & \frac{G_{F} h}{\sqrt{2}} \frac{M^{6}}{256 \pi^{6}} L^{-\frac{4}{9}} \times \\
& \frac{1}{36}\left[-118 E_{2}\left(\frac{W}{M}\right)+24 F_{2}\left(\frac{W}{M}, \frac{M}{\bar{\mu}}\right)\right]\left(\frac{1}{3} \sin ^{2} \theta_{W}\right)
\end{aligned}
$$




$$
\begin{aligned}
h_{\rho}^{2}= & 0 \\
\frac{h_{\rho}^{1^{\prime}}}{2 m} \frac{\lambda_{N}^{2}}{M^{2}} e^{-\frac{m^{2}}{M^{2}}=} & -\frac{G_{F} h}{\sqrt{2}} \chi\langle\bar{q} q\rangle \frac{M^{4}}{432 \pi^{4}} L^{-\frac{16}{27}} \times \\
& {\left[-13 E_{1}\left(\frac{W}{M}\right)+6 F_{1}\left(\frac{W}{M}, \frac{M}{\bar{\mu}}\right)\right]\left(\frac{1}{3} \sin ^{2} \theta_{W}\right) } \\
\frac{\lambda_{N}^{2} h_{\omega}^{0}}{M^{2}} e^{-\frac{m^{2}}{M^{2}}=} & -\frac{G_{F} h}{\sqrt{2}} \frac{M^{6}}{256 \pi^{6}} L^{-\frac{4}{9}} \times \\
\frac{\lambda_{N}^{2} h_{\omega}^{1}}{M^{2}} e^{-\frac{m^{2}}{M^{2}}=}= & -\frac{G_{F} h}{\sqrt{2}} \frac{M^{6}}{256 \pi^{6}} L^{-\frac{4}{9}} \times \\
& \frac{5}{36}\left[-70 E_{2}\left(\frac{W}{M}\right)+24 F_{2}\left(\frac{W}{M}, \frac{M}{\bar{\mu}}\right)\right]\left(\frac{1}{3} \sin ^{2} \theta_{W}\right)
\end{aligned}
$$

The function $L\left(\equiv \ln \left(M / \Lambda_{Q C D}\right) / \ln \left(\bar{\mu} / \Lambda_{Q C D}\right)\right.$ with $\Lambda_{Q C D}=100 \mathrm{MeV}$ and $\bar{\mu}=$ $0.5 \mathrm{GeV}$ ) is introduced to take care of the anomalous dimensions. Unlike our analysis of the QCD sum rules for the strong couplings $g_{\rho}$ and $g_{\omega}$ [7], loop integrations in the weak parity-violating case yields subdivergences in the form of $p^{2 n}\left[\ln \left(-p^{2} / \bar{\mu}^{2}\right)\right]^{2}$ with $n$ some integer which, upon Borel transform, yield terms proportional to $M^{2 n-2} \ln \left(M^{2} / \bar{\mu}^{2}\right)$ in the QCD sum rules. Thus, the value for the dimensional parameter $\bar{\mu}$ is of some numerical importance. The standard choice $\bar{\mu}=0.5 \mathrm{GeV}$ is used in this paper.

Furthermore, the quantity $W$ (with the standard choice of $W=1.45 \mathrm{GeV}$ ) is the threshold used in the continuum approximation, in which the contributions due to the excited states and the continuum are approximated by what may be obtained at the quark level (by use of QCD). The continuum approximation introduces, into the sum rules (20)-(25), the functions $E_{i}$ and $F_{i}$ which are defined in the Appendix.

We should also mention that the QCD sum rules which we have obtained for PV couplings are basically those of leading order in QCD (with the nontrivial vacuum structure). It is a tremendous task to try to include a sufficient number of terms which involves the condensates of higher dimension in nature. Nevertheless, it is important to emphasize that the QCD sum rule approach is a definitive (deductive) procedure for evaluating the various diagrams based on QCD, contrary to the qualitative or semiquantitative nature of the earlier DDH approach [3]. In other words, the present QCD sum approach can be improved upon, albeit fairly complicated, order in order in QCD and with increasing dimensions.

\section{$3 \quad$ Numerical analysis}

The input parameters for our numerical analysis are those commonly adopted in standard QCD sum rule analyses [7, 6, 8, 10]. 


$$
\begin{array}{lll}
\lambda_{N}^{2}=1.2 \times 10^{-3} \mathrm{GeV}^{6}, & a=0.546 \mathrm{GeV}^{3}, & b=0.47 \mathrm{GeV}^{4} \\
& \chi=-6 \mathrm{GeV}^{-2}, & m_{0}^{2}=0.8 \mathrm{GeV}^{2} \\
h=4.65, & g_{\rho}=2.79, & g_{\omega}=8.37
\end{array}
$$

Variations in some of these parameter values may result in errors of numerical importance. Neglect of higher dimensional terms brings in uncertainties which are difficult to quantify (unless some such terms can explicitly be taken into account). Nevertheless, we may use our experience from analyzing other QCD sum rules, such as those for the strong $\rho N N$ and $\omega N N$ couplings [7], to assess these errors.

Before performing the standard numerical analysis, we wish to first use the QCD sum rules (20)-(25), together with some data from the nuclear parity violation experiments, to provide some estimates on the various parity-violating (PV) couplings. We note that both the couplings $h_{\rho}^{0}$ and $h_{\omega}^{0}$ receive contributions from both $W^{ \pm}$and $Z^{0}$ exchanges while other PV couplings are dictated by $Z^{0}$ exchange. As a result, the couplings $h_{\rho}^{0}$ and $h_{\omega}^{0}$ are much larger than the other PV coupling constants, which are suppressed by a factor $(1 / 3) \sin ^{2} \theta_{W}=0.07$. This means that, as an approximation, we may neglect the couplings $h_{\rho}^{1}, h_{\rho}^{2}, h_{\rho}^{\prime 1}$ and $h_{\omega}^{1}$. Another important approximate relation is $h_{\rho}^{0}=h_{\omega}^{0}$.

There are many parity violation measurements in processes involving complex nuclei, leading to the determination of the quantity $X_{N}^{P}$, which characterizes the strength of PV interaction. This quantity, with an experimental value of about $3 \times$ $10^{-6}[13$, can be expressed in terms of the PV coupling constants:

$$
X_{N}^{P}=5.5 f_{\pi}-0.25 g_{\rho} h_{\rho}^{1}-0.62 g_{\rho} h_{\rho}^{0}-0.05 g_{\rho} h_{\rho}^{\prime 1}-0.17 g_{\omega} h_{\omega}^{1}-0.19 g_{\omega} h_{\omega}^{0} .
$$

The PV $\pi N N$ coupling $f_{\pi}$ may be obtained via the QCD sum rule method and it is about $(3.0 \pm 0.5) \times 10^{-7}\left[14\right.$, 10]. Thus, we have $h_{\rho}^{0} \approx h_{\omega}^{0} \approx-4.1 \times 10^{-7}$, by assuming that the other PV couplings $\left(h_{\rho}^{1}, h_{\rho}^{2}, h_{\rho}^{\prime 1}, h_{\omega}^{1}\right)$ are considerably smaller in comparison.

Analogously, we may also use the expression of the asymmetry $A_{p p}$, as observed in polarized proton-proton scattering, to determine the PV couplings:

$$
A_{p p}(15 M e V)=0.01 g_{\omega}\left(h_{\omega}^{0}+h_{\omega}^{1}\right)+0.03 g_{\rho}\left(h_{\rho}^{0}+h_{\rho}^{1}+\frac{h_{\rho}^{2}}{\sqrt{6}}\right),
$$

with an experimental value of $-(1.7 \pm 0.85) \times 10^{-7}[3$. It yields a value of about $-(10 \pm 5) \times 10^{-7}$ for $h_{\rho}^{0}$ and $h_{\omega}^{0}$, the magnitude of which is somewhat larger than the previous value but remains consistent in light of large errors.

We proceed to analyze the various QCD sum rules as a function of the Borel mass squared $M^{2}$. The choice of the range for the Borel mass is guided by the study of the QCD sum rules for other properties of the nucleon, such as the nucleon mass, the magnetic moments, the axial couplings, or the strong and weak $\pi N N$ couplings. Specifically, it has been found in general that the nucleon sum rules should give rise to reasonable predictions in the Borel mass range of $0.9 \mathrm{GeV}^{2} \leq M^{2} \leq 1.1 \mathrm{GeV}^{2}$. Such choice of the Borel mass squared also enable us to estimate the errors of our 
QCD sum rule predictions by inferring from the analysis of the other QCD sum rules for the nucleon. The error could be as large as about $25 \%$ in some special cases.

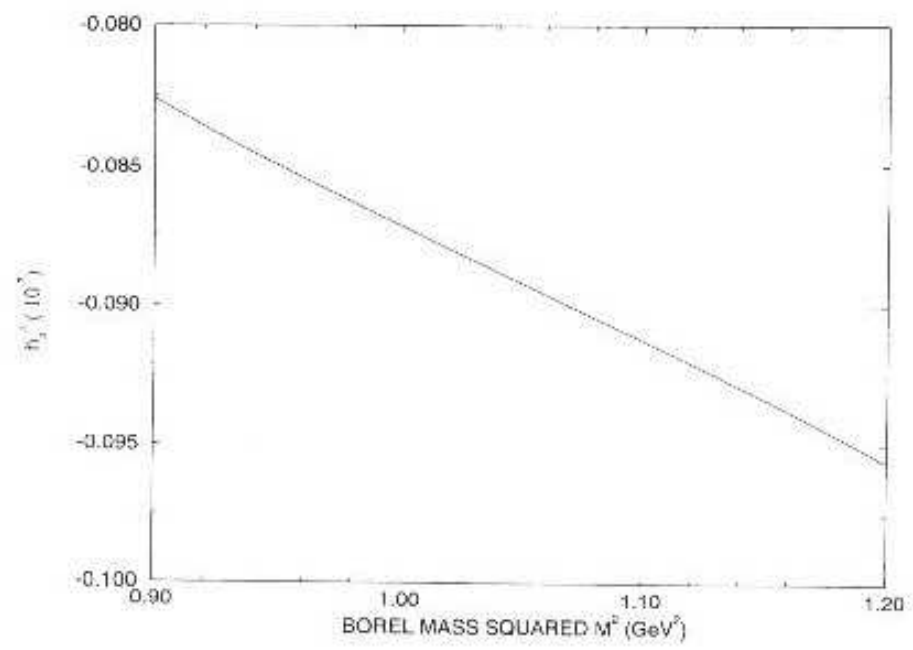

Figure 2: The PV coupling $h_{\rho}^{0}$ (or $h_{\omega}^{0}$ ) is plotted as a function of the Borel mass squared $M^{2}$ in the range of $(0.9-1.2) \mathrm{GeV}^{2}$.

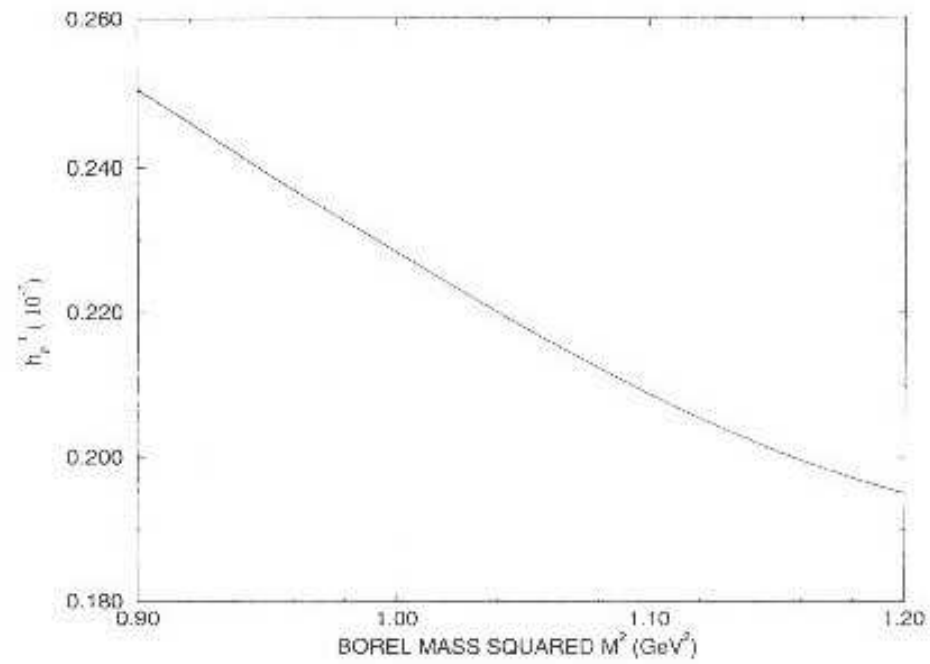

Figure 3: The PV coupling $h_{\rho}^{1}$ is plotted as a function of the Borel mass squared $M^{2}$ in the range of $(0.9-1.2) \mathrm{GeV}^{2}$.

In Figs. 2-5, the various couplings are shown as a function of the Borel mass squared $M^{2}$ (in units of $G e V^{2}$ ). It is of some importance to note that the scales in these figures are in fact different, resulting in errors of different magnitudes. As long as we have faith in these sum rules when a sufficient number of higher dimensional 
terms are included, it makes sense to make (semi-quantitative) predictions with the aid of only a couple of leading terms. The following predictions have been obtained in this way:

$$
\begin{array}{cc}
h_{\rho}^{0}: & -(6.9 \pm 3.6) \times 10^{-7} \\
h_{\rho}^{1}: & -(0.087 \pm 0.004) \times 10^{-7} \\
h_{\rho}^{2}: & 0 \\
h_{\rho}^{1^{\prime}}: & (0.23 \pm 0.02) \times 10^{-7} \\
h_{\omega}^{0}: & -(6.9 \pm 3.6) \times 10^{-7} \\
h_{\omega}^{1}: & -(0.03 \pm 0.04) \times 10^{-7} .
\end{array}
$$

The large errors, such as those associated with $h_{\rho}^{0}$ and $h_{\omega}^{0}$, are caused primarily by the rapid variation of the prediction in the quoted Borel mass range. The experience with the QCD sum rule method, such as in the weak parity-violating coupling $f_{\pi N N}$ [10], suggests that inclusion of the next couple of terms of higher dimensions could help to smoothen the rapid-varying behavior of the leading term while yielding predictions in the same ballpark.

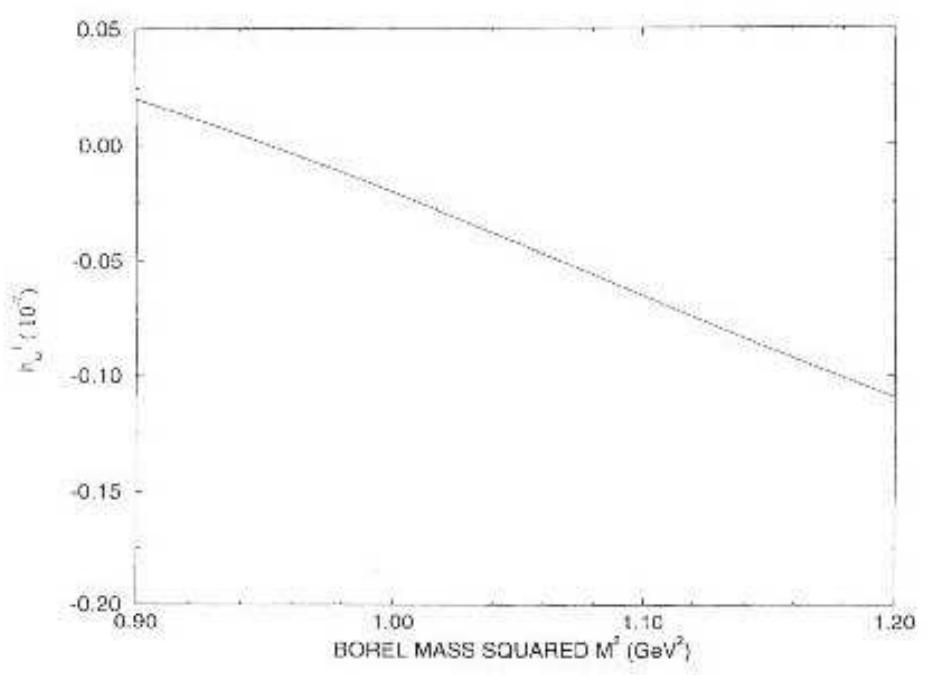

Figure 4: The PV coupling $h_{\rho}^{\prime 1}$ is plotted as a function of the Borel mass squared $M^{2}$ in the range of $(0.9-1.2) \mathrm{GeV}^{2}$.

We note that the predicted values for $h_{\rho}^{0}$ and $h_{\omega}^{0}$ are compatible with the above results as extracted from the experimental values of $X_{N}^{P}$ and $A_{p p}(15 \mathrm{MeV})$ (together with the observation from the QCD sum rules that only $h_{\rho}^{0}$ and $h_{\omega}^{0}$ are dominant).

In Table I, we compare the various predictions on parity-violating meson-nucleon couplings, including the estimates given by Desplanques, Donoghue, and Holstein 


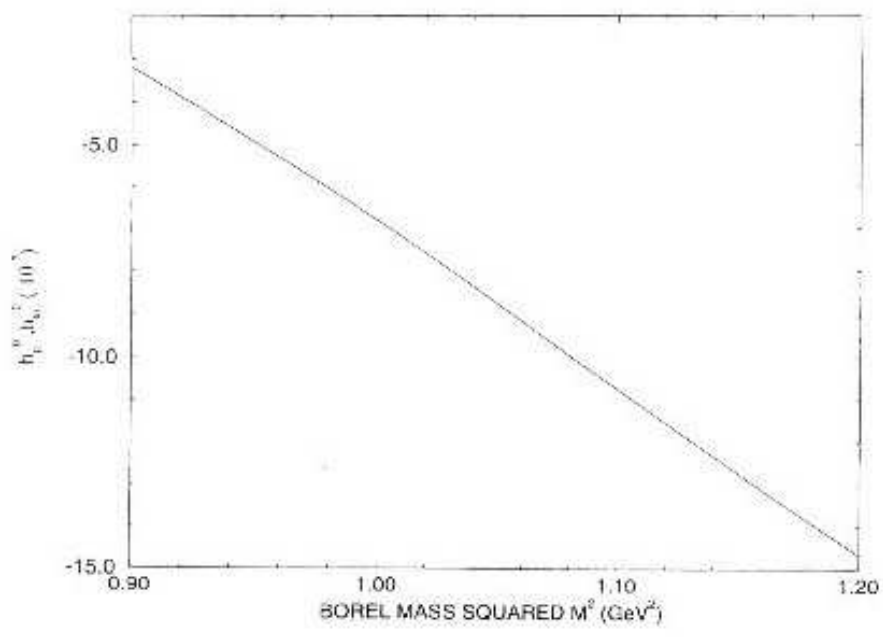

Figure 5: The PV coupling $h_{\omega}^{1}$ is plotted as a function of the Borel mass squared $M^{2}$ in the range of $(0.9-1.2) \mathrm{GeV}^{2}$.

\begin{tabular}{|l|l|l|l|}
\hline & DDH (best estimate) & AH (best fit) & QCD sum rules \\
\hline$f_{\pi}$ & $0 \rightarrow 11(4.6)$ & $0 \rightarrow 11(2.1)$ & $3.0 \pm 0.5^{*}$ \\
\hline$h_{\rho}^{0}$ & $-31 \rightarrow 11(-11.4)$ & $-31 \rightarrow 11(-5.8)$ & $-(6.9 \pm 3.7)$ \\
\hline$h_{\rho}^{1}$ & $-0.4 \rightarrow 0(-0.19)$ & $-0.5 \rightarrow 0.4(-0.22)$ & $-(0.087 \pm 0.004)$ \\
\hline$h_{\rho}^{2}$ & $-7.6 \rightarrow-11(-9.5)$ & $-6.3 \rightarrow-10(-7.1)$ & 0 \\
\hline$h_{\rho}^{\prime 1}$ & 0 & 0 & $0.23 \pm 0.02$ \\
\hline$h_{\omega}^{0}$ & $-10 \rightarrow 5.7(-1.9)$ & $-12 \rightarrow 2.6(-5.0)$ & $-(6.9 \pm 3.7)$ \\
\hline$h_{\omega}^{1}$ & $-0.8 \rightarrow-1.9(-1.1)$ & $-3.1 \rightarrow-1.1(-2.4)$ & $-(0.03 \pm 0.04)$ \\
\hline
\end{tabular}

Table 1: PV couplings in units of $10^{-7}$. The numbers in the brackets are the "best" values. ${ }^{*}$ The prediction is obtained from [14].

(DDH) 3, the values by an overall fit to the existing experiments (AH) 4], and ours making use of the QCD sum rules. Our results, if taken seriously, sharpen the allowed ranges for most of the six couplings. The overall agreement is good, although we should note that, from the leading diagrams which we have included, the isotensor coupling $h_{\rho}^{2}$ vanishes (while it fairly sizable in [3, 4]) and $h_{\rho}^{\prime 1}$ is small but is different from zero (as in [3, 4]). [Both $h_{\rho}^{2}$ and $h_{\rho}^{\prime 1}$ do not contribute in any major way to the existing nuclear parity violation observables. See, for example, the expressions for $X_{N}^{P}$ and $\left.A_{p p}(15 \mathrm{MeV}).\right]$

Owing to the tremendous complications to include a sufficient number of higher dimensional terms involving the various condensates, we have relied on the experience of analyzing the other QCD sum rules for the nucleon in order to assess the uncertainties. While it is clearly desirable to improve on these derivations (by including more higher order terms, especially for $h_{\rho}^{0}$ and $h_{\omega}^{0}$ ), it is indeed gratifying to note that our overall predictions are fairly consistent with earlier results [3, 4]. 


\section{Summary}

The problem of parity-violating nuclear force has been a very difficult one. Progresses have been made in the past [3, 4] in the simple meson-exchange picture, in which parity-violating nuclear force arises from exchange of $\pi, \rho, \omega$, or other meson with strong $M N N$ coupling at one vertex and weak parity-violating $M N N$ coupling at the other vertex. The QCD sum rule method allows for a determination of the various parity-violating $M N N$ couplings starting from QCD (with the nontrivial vacuum) and Glashow-Salam-Weinberg (GSW) electroweak theory. I believe that the QCD sum rule methods, which make explicit connection between the underlying theory (i.e. the QCD and GSW electroweak theory) and the predictions, offer us a systematic method to tackle. We wish to note that our QCD sum rule predictions are fairly consistent with earlier results [3, 4, 5].

\section{Acknowledgments}

The authors wish to acknowledge Center for Theoretical Physics of MIT for the hospitalities extended to them during which the major part of this project was carried out. This work was supported by the National Science Council of R.O.C. (through the grant NSC96-2112-M002-023-MY3 and NSC96-2752-M002-007-PAE).

\section{Appendix: Borel Transformation}

For the invariant functions $f\left(p^{2}\right)$ appearing in the polarization function, we may apply the following dispersion relation,

$$
f(s)=\frac{1}{\pi} \int_{0}^{\infty} \frac{\operatorname{Im} f\left(p^{2}\right)}{p^{\prime 2}+s} d p^{\prime 2}, \quad s=-p^{2},
$$

with a necessary number of subtractions.

Borel transformation is a general tool employed in the method of QCD sum rules. It is defined by

$$
B f(s)=\lim _{\substack{n, s \rightarrow \infty \\ s / n=M^{2}}} \frac{s^{n+1}}{n !}\left(-\frac{d}{d s}\right)^{n} f(s)
$$

Apply Borel transformation to the dispersion relation we obtain

$$
B f(s)=\frac{1}{\pi} \int_{0}^{\infty} e^{-p^{2} / M^{2}} \operatorname{Im} f\left(p^{2}\right) d p^{2} .
$$

In general, we may take into account the contribution due to exited states and assume that it is equal to the quark-level contribution of $f\left(p^{2}\right)$ starting from some 
cutoff value $p^{2}=W^{2}$. That is, we use a modified Borel transformation $B_{W}$ with the definition:

$$
B_{W} f(s)=\frac{1}{\pi} \int_{0}^{W^{2}} e^{-p^{2} / M^{2}} \operatorname{Im} f\left(p^{2}\right) d p^{2}
$$

which involves some cutoff $W$.

We list all the modified Borel transformation functions which we need in the text.

$$
\begin{aligned}
& -M^{2} E_{0}\left(\frac{W}{M}\right) \equiv B_{W} \ln \frac{s}{\mu^{2}} \\
& =-M^{2}\left(1-e^{-W^{2} / M^{2}}\right) \\
& M^{4} E_{1}\left(\frac{W}{M}\right) \equiv B_{W} s \ln \frac{s}{\mu^{2}} \\
& =M^{4}\left[1-e^{-W^{2} / M^{2}}\left(1+\frac{W^{2}}{M^{2}}\right)\right] \\
& -2 M^{6} E_{2}\left(\frac{W}{M}\right) \equiv B_{W} s^{2} \ln \frac{s}{\mu^{2}} \\
& =-2 M^{6}\left[1-e^{-W^{2} / M^{2}}\left(1+\frac{W^{2}}{M^{2}}+\frac{W^{4}}{2 M^{4}}\right)\right] \\
& 2 M^{4} F_{1}\left(\frac{W}{M}, \frac{M}{\mu}\right) \equiv B_{W} s \ln ^{2} \frac{s}{\mu^{2}} \\
& =2 M^{4}\left\{1-\gamma-e^{-W^{2} / M^{2}}+E i\left(-\frac{W^{2}}{M^{2}}\right)\right. \\
& \left.+\left[1-e^{-W^{2} / M^{2}}\left(1+\frac{W^{2}}{M^{2}}\right)\right] \ln \frac{M^{2}}{\mu^{2}}\right\} \\
& -4 M^{6} F_{2}\left(\frac{W}{M}, \frac{M}{\mu}\right) \equiv B_{W} s^{2} \ln ^{2} \frac{s}{\mu^{2}} \\
& =-4 M^{6}\left\{\frac{3}{2}-\gamma-\frac{3}{2} e^{-W^{2} / M^{2}}\left(1+\frac{W^{2}}{3 M^{2}}\right)+E i\left(-\frac{W^{2}}{M^{2}}\right)\right. \\
& \left.+\left[1-e^{-W^{2} / M^{2}}\left(1+\frac{W^{2}}{M^{2}}+\frac{W^{4}}{2 M^{4}}\right)\right] \ln \frac{M^{2}}{\mu^{2}}\right\}
\end{aligned}
$$

Here we have adopted the conventional symbols $E_{0}, E_{1}$ and $E_{2}$, and introduced two new symbols $F_{1}$ and $F_{2}$. The exponential integral function $\operatorname{Ei}(x)$ is defined by

$$
\operatorname{Ei}(x)=-\int_{-x}^{\infty} \frac{e^{-t}}{t} d t
$$

\section{References}

[1] W.T.H. van Oers, private communication. 
[2] J. Carlson, R. Schiavilla, V.R. Brown, B.F. Gibson, C65, 035502-1 (2002).

[3] B. Desplanques, J.F. Donoghue, and B.R. Holstein, Ann. Phys. (New York) 124, 449 (1980).

[4] E.G. Adelberger and W.C. Haxton, Ann. Rev. Nucl. Part. Sci. 35, 501 (1985).

[5] M.J. Ramsey-Musolf and S.A. Page, Ann. Rev. Nucl. Part. Sci. 56, 1 (2006).

[6] M.A. Shifman, A.I. Vainshtein and V.I. Zakharov, Nucl. Phys. B147, 385, 448 (1979).

[7] Chih-Yi Wen and W-Y. P. Hwang, Phys. Rev. C56, 3346 (1997).

[8] B.L. Ioffe and A.V. Smilga, Nucl Phys. B232, 109 (1984); B. L. Ioffe, Z. Phys. C18, 67 (1983).

[9] B.L.Ioffe, Z. Phys. C18, 67 (1983).

[10] E.M. Henley, W-Y. P. Hwang, and L.S. Kisslinger, Phys. Lett. B367, 21 (1996); Addendum, B440, 449 (1998).

[11] W-Y. P. Hwang, Ze-sen Yang, Y.S. Zhong, Z.N. Zhou, and Shi-lin Zhu,Phys. Rev. C57, 61 (1998).

[12] E.M. Henley, W-Y. P. Hwang, and L.S. Kisslinger, manuscript in preparation.

[13] B. Desplanques and J. Missimer, Nucl Phys. A300, 286 (1978).

[14] W-Y. P. Hwang, "QCD sum rules and chiral symmetry breaking", Preprint MITCTP-2498 \& hep-ph/9601219, Z. Phys. C75, 701 (1997). 